\title{
MANAGING RISK IN FORCE DEVELOPMENT PROGRAMS
}

\section{Venelin GEORGIEV}

\begin{abstract}
Examining the dynamic character of the Armed Forces transformation, viewed as a continuous process for development and adaptation of the security policy to the external environment by implementing the Force Development Programs in order to improve the Armed Forces operational capabilities, makes it possible to determine some main characteristics - necessity of continuous management including effective planning, efficient organization, and realization and reliable control of the achieved results; management in conditions of uncertainty, i.e. in conditions of lack of complete information about the different parts of the whole process and the external environment, in which this process will be implemented. The efficiency of the risk management process for the Force Development Programs depends on different factors, but the most significant role belongs to the structure of the process and the methods used in the risk management model. The structure of the risk management process determines the comprehensive, systematic and complex character of the model and the influence of the methods on the quality of the results achieved by the model used in the decision-making process in the area of Armed Forces transformation and Force Development Programs implementation.
\end{abstract}

Keywords: Armed Forces transformation, force development program, risk and risk management, risk identification, risk analysis, risk mitigation, risk tracking, sensitive analysis, decision tree.

The examination on the dynamic character of the Armed Forces transformation, viewed as a continuous process of development and adaptation of the security policy to the external environment by implementing the Force Development Programs in order to improve the Armed Forces operational capabilities helps in determining some main characteristics.

The first characteristic of the Armed Forces transformation process is the necessity of continuous management including effective planning, efficient organization, and realization and reliable control of the achieved results. Having such a process that can be managed effectively is one of the essential conditions for achieving the final results and the desired end state in the area of Armed Forces transformation. 


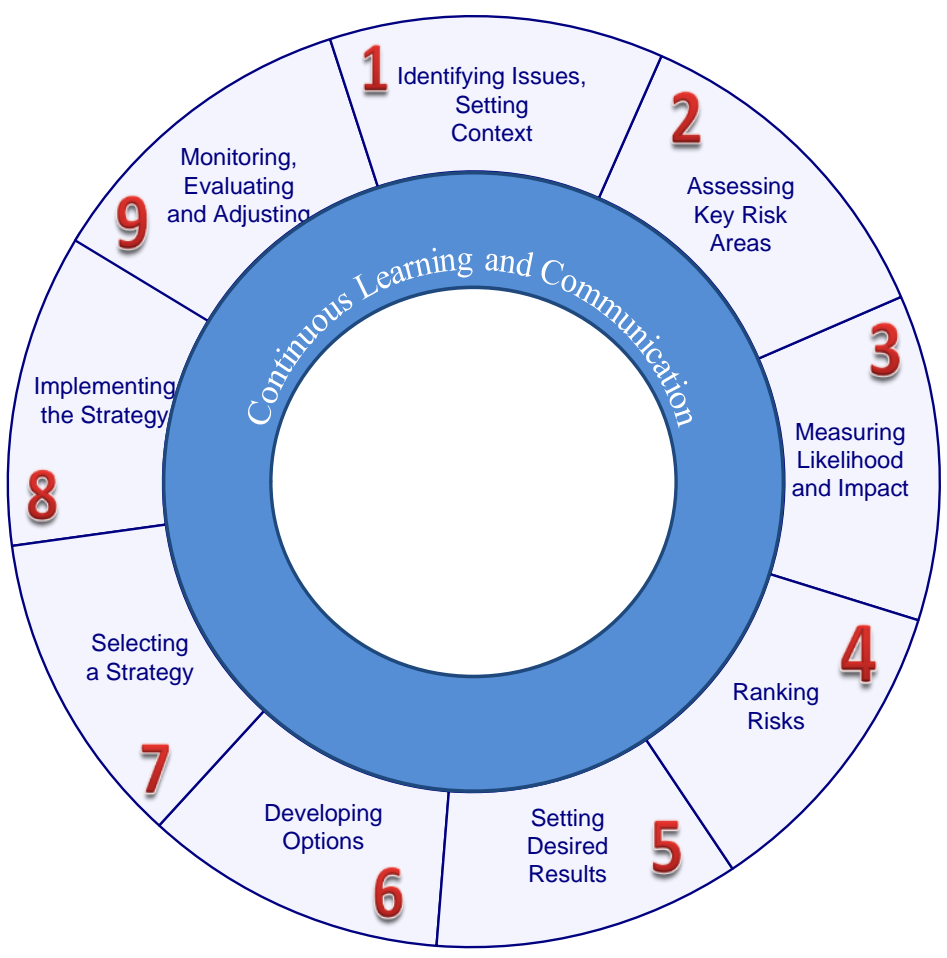

Figure 1: Risk Management Process - Model 1.

The second characteristic is that the process of Armed Forces transformation can be viewed as management in conditions of uncertainty, i.e. in conditions of lack of complete information about the different parts of the whole process and the external environment, in which this process will be implemented. The existing uncertainty is a source of different types of risk that have to be identified, estimated, analyzed and mitigated during planning and implementation of the transformation activities within the Force Development Programs. The significance of the Armed Forces transformation process and the variety of present risks - political, organizational, technological, financial, etc.-impose the necessity to implement a risk management process as an element of the Armed Forces transformation process and the Force Development Programs implementation.

The efficiency of the risk management process for the Force Development Programs depends on different factors, but the most significant role is assigned to the structure of the process and the methods used within the risk management model. The structure of the risk management process determines the comprehensive, systematic and comp- 


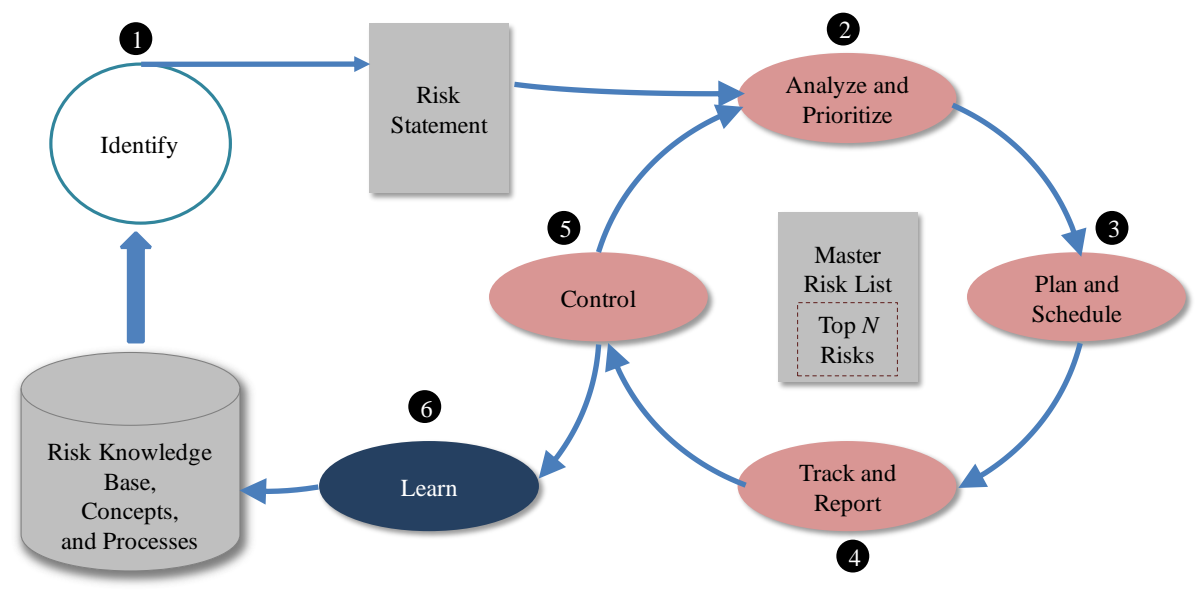

Figure 2: Risk Management Process - Model 2.

lex character of the model and the influence of the methods on the quality of the results, achieved by the model used in the decision-making process in the area of Armed Forces transformation and Force Development Programs implementation.

The definition of a rational model of the risk management process is connected with analysis of the existing models for implementation of this process and development of a new one that is suitable for the Armed Forces transformation process. In the theory and practice of risk management there are many examples of models of risk management processes, which will be presented in what follows.

Figure 1 illustrates a model of the risk management process that includes nine main elements. ${ }^{1}$ The advantages that the implementation of this model brings could be summarized as follows. The model makes it possible to:

- Define the likely problems of the implementation of the Force Development Programs, their scope, content and the existing feasible alternatives for their solution.

- Perform qualitative and quantitative assessment of the identified program risks by using the probabilities for their realization and the expected results.

- Rank the estimated risks using the existent or novel models, methods and criteria.

- Create, choose and implement suitable strategies for risk mitigation by reducing the risk probabilities and the expected results.

- Develop and implement the risk management and risk mitigation plans. 


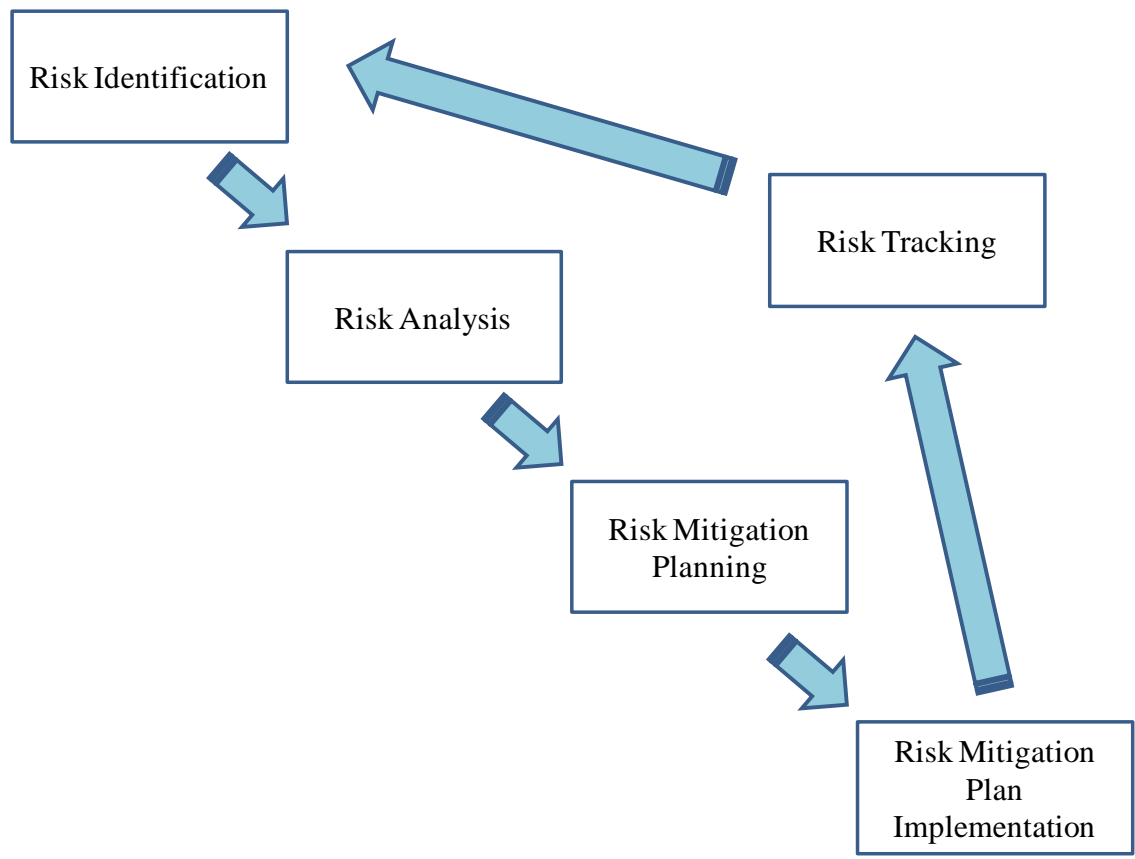

Figure 3: Risk Management Process - Model 3.

- Document and report the result from the risk management process and take the necessary measures for improvement of the process in order the desired results to be achieved.

Figure 2 presents a second model of the risk management process, which includes six elements. ${ }^{2}$

According to the second model, risk identification is a continuous process that has to begin as early as possible and has to be repeated with appropriate frequency during the period of program implementation. Risk analysis is presented as transformation of a database into specific forms that are used in the process of risk prioritization. The model includes the development of a risk management framework that makes it possible to implement the risk management activities as part of the day-to-day work. The purpose of the last element of the model is to define the lessons learned for the Force Development Programs risk management process, which could be used in the future for management of similar programs.

Figure 3 illustrates a third model of the risk management process, which is taken from the Defense Acquisition Guidance and includes five elements. ${ }^{3}$ The individual risk 


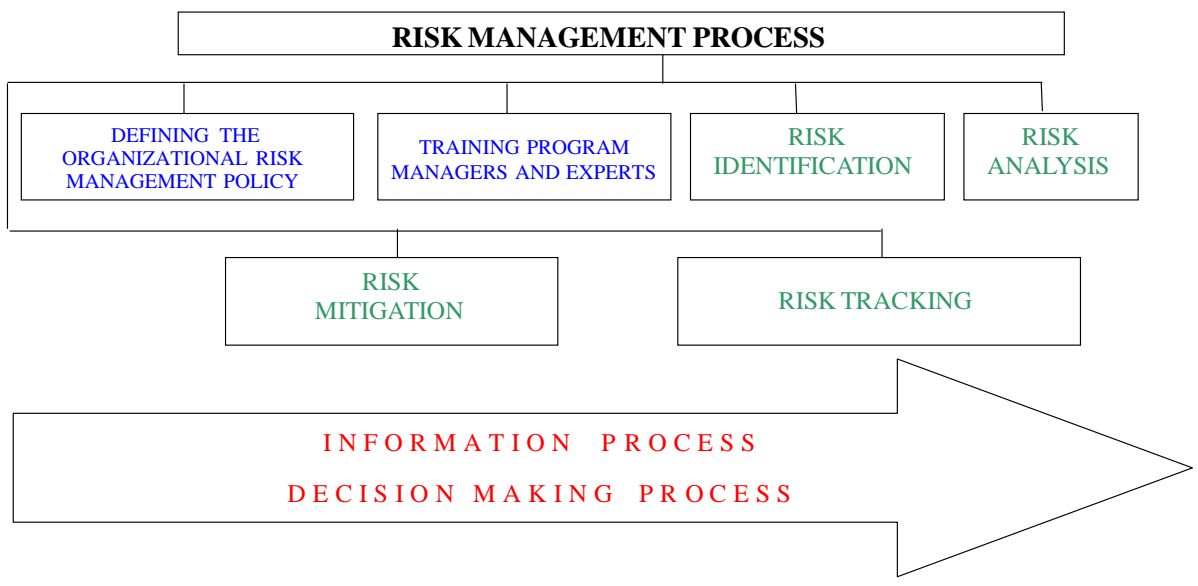

Figure 4: A Risk Management Process that Is Used in the MoD Practice.

management activities are given in a more general way as risk identification, risk analysis, risk mitigation planning, risk mitigation plan implementation and risk tracking.

The analysis of the different alternatives for models of the risk management process enables to conclude that their content is quite similar and that the main reason for the existing differences is the level of comprehensiveness used to describe the process of risk management.

Figure $4^{4}$ presents the model of the process of risk management that is used in the practice of the Bulgarian Ministry of Defense (MoD). The structure of the model is similar to the previous three models and reflects some specific characteristics of the process of implementation of the Force Development Programs. This model includes eight elements that could be divided into six phases and two processes as follows:

- Main phases or phases directly connected with the risk management process. These phases include risk identification, risk analysis, risk mitigation and risk tracking.

- Supporting phases that support the implementation of the main phases of the process. They are connected with the organizational risk management policy and could be defined as establishing an organizational risk management policy, defining the rights and responsibilities of the participants in the process and training of program managers and experts who will work in the area of risk management during the Force Development Programs implementation. 
- Supporting processes that support all activities included in the risk management model and that could be defined as decision-making process and information process.

The content of each main phase of the risk management model could be described as follows. ${ }^{5}$

\section{Risk Identification}

It gives an answer to the question "What can go wrong with the implementation of the Force Development Programs?”

As an activity, risk identification has to begin as early as possible and it lasts throughout the program with regular reviews and analysis of the performance, schedule, budget, etc.

Risk identification is accomplished by means of appropriate methods through program decomposition into relevant program elements or areas, which provides an opportunity the sources of program risk to be well studied.

\section{Risk Analysis}

It gives an answer to the question "How big is the risk?"

Each undesirable event that might affect the success of the program should be assessed as to likelihood and consequence of occurrence.

Risk analysis is accomplished after program risk identification using appropriate methods.

\section{Risk Mitigation}

It gives an answer to the question "What is the program approach for addressing the potential unfavorable consequence?"

Based on the assessments from the previous phase, risk mitigation is performed on the identified program risks with high level of significance.

Risk mitigation is achieved defining and using appropriate strategies.

\section{Risk Tracking}

It gives an answer to the question "How are things going?"

Risk tracking activities are integral to good program management and provide much of the information used to identify any performance, schedule, readiness and cost barriers to meeting program objectives. 


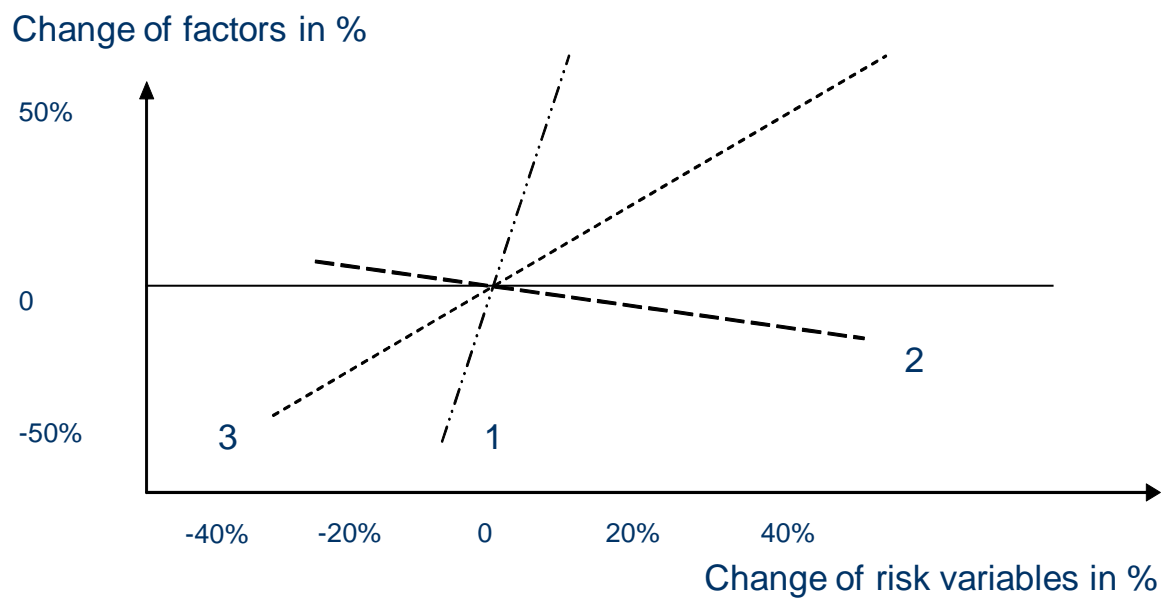

Figure 5: Sensitivity Analysis of Program Risks.

Risk tracking is accomplished by means of appropriate methods for control of the current status of the program.

The efficiency of the risk management process of the Force Development Programs depends on the methods used in the different phases described above. Equally suitable for the risk management practice in the Force Development Programs implementation could not only be the traditional methods but also some of the new approaches. Depending on the specific characteristics of the program and the concrete phase of the implementation process, it is right of the managers and experts to decide which method to be used in practice.

Different methods could be used for risk identification in the Force Development Programs, with proven efficiency. These methods could be divided into two main groups - methods for expert assessment and methods for physical identification of the program risk.

The review of the MoD practice in the area of program risk identification shows that the most frequently used methods for expert assessment are the Delphi method, Brainstorming and the method based on developed scenarios. These methods make it possible to collect and use the opinion of different experts in order to identify the program risk in a scientific way.

Methods for physical identification of the program risks are also used in the MoD practice, but not so often. They play supplementary role in the process of program 
risk identification. These methods are based on the information from the program documents and they are with high level of accuracy.

Depending on the specific characteristics of the programs, different methods could be used for qualitative and quantitative analysis of program risk. For example:

- The Matrix Method is a method that could be used for simple and small programs. The matrix includes the probability of the identified risk events and one of the main parameters of the program, such as schedule, budget, scope, quality, etc. This method is not very precise and this is the reason to be not so frequently used for large and complex programs.

- The Statistical Method is one of the most frequently applied methods for program risk analysis. It is a method with high level of accuracy and it is based on mathematical computations.

- Sensitivity Analysis is defined as the most accessible method for program risk analysis. It makes it possible to identify the risk events that have a significant influence on program implementation. Figure 5 demonstrates the results from the application of sensitivity analysis where it is visible that program implementation depends to a great extent on risk event 1 and that it is not so sensitive to factors 2 and 3 .

- $\quad$ The Decision Tree is a graphical method for analysis that presents the possible courses of actions in Defense Development Programs implementation and the expected results (see Figure 6). This method is frequently applied in the analysis of program costs.

In conclusion, we could summarize that the significance of the goals of the Armed Forces transformation process necessitates the application of a risk management pro-

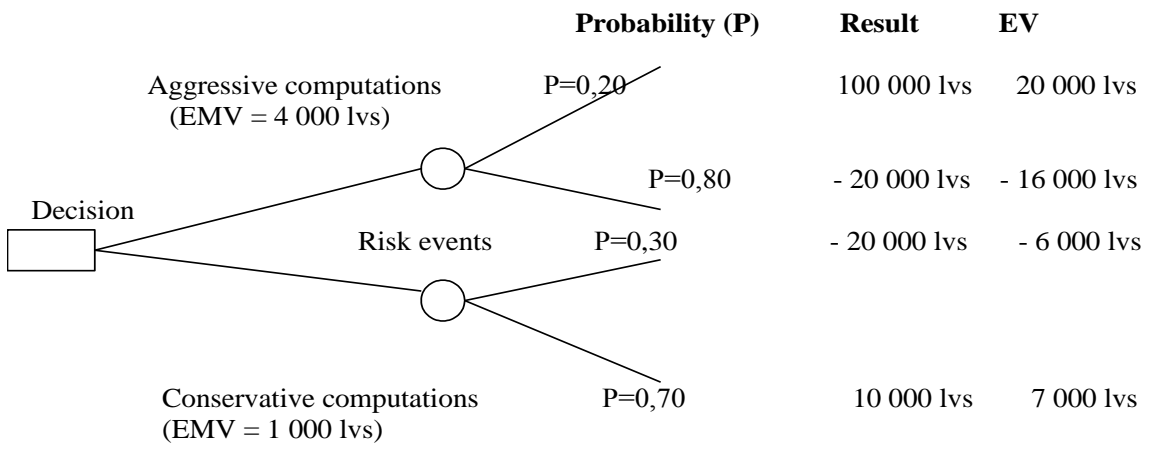

Figure 6: A Decision Tree. 
cess for the Defense Development Program implementation as part of the whole management process. The effects from program risk management depend on the model of the process and the applied methods. The model and the applied methods should not only reflect the best practices; they have to be also suitable for managing the Defense Development Programs in the current security environment. The program teams should be aware of the fact that there are no universal models and methods that could be used in any case and in any program. The choice of appropriate models and methods for program risk management is an activity that requires considerable knowledge and experience and the program managers and experts are the people who decide on the exact models and methods to be used in order to achieve the program goals.

\section{Notes:}

1 Integrated Risk Management Framework (Treasury Board of Canada, April 2001), <www.tbs-sct.gc.ca/pubs_pol/dcgpubs/RiskManagement/dwnld/rmf-cgr_e.pdf> (23 April 2008).

2 The Microsoft ${ }^{\circledR}$ Operations Framework (MOF) Risk Management Discipline for Operations, Updated February 19, $2004<$ http://www.microsoft.com/technet/solutionaccelerators/ its/mo/mof/mofrisk.mspx> (23 April 2008).

${ }^{3}$ Risk Management Guide for DoD Acquisition, Sixth Edition (Version 1.0), (Washington DC: Office of the Under Secretary of Defense for Acquisition Technology and Logistics, August 2006), <http://www.sei.cmu.edu/programs/acquisition-support/publications/dodriskguide.pdf> (23 April 2008).

${ }^{4}$ Venelin Georgiev, Risk Management (Sofia: Voenno Izdatelstvo, 2005).

${ }^{5}$ Risk Management Guide for DoD Acquisition.

Colonel VENELIN GEORGIEV is a state expert in Defense Acquisition Policy Department in Armament and Equipment Policy Directorate, MoD. He holds a doctoral degree in the area of management and master degrees in the areas of technical, military and economic sciences. His experience is in the areas of program management of the defense resources, project management, defense acquisition and risk management. He is author of more than 50 articles in the area of Defense and Armed Forces management. 